\title{
Avaliação do nível de conhecimento e motivação relativo à saúde bucal em duas escolas de Londrina, Paraná
}

\author{
Evaluation of the knowledge level and motivation \\ related to the oral health in \\ two schools of Londrina, State of Paraná
}

Adriana Yumi Miyazaki*

Fábio J.A.R. Takushi*

Mauri Reiche**

MIYAZAKI, A.Y.; TAKUSHI, F.J.A.R.; REICHE, M. Avaliação do Nível de Conhecimento e Motivação Relativo à Saúde Bucal cm duas Escolas de Londrina, Paraná. Seminina, Londrina, v. 18, ed. especial, p. 83 - 89, fev. 1997.

RESUMO: O presente trabalho refere-se a uma pesquisa de campo para avaliar o nivel de conhecimento e motivação relativo à saúde bucal em crianças de duas escolas da cidade de Londrina, Paraná: "Colégio Universitário" (Escola 1) e "Colégio Estadual Olympia M. de Tormenta" (Escola 2), onde foi analisada, em cada escola, a importância que as crianças davam à saúde bucal e o nivel de informações sobre a higiene e os meios de prevenção da cárie dentária. Os dados foram coletados através de questionários aplicados a 168 escolares de ambos sexos, sendo 86 de cada escola, com idade variando de 11 a 15 anos (média de idade $=12$ anos). A análise dos resultados obtidos permitiu concluir que o nivel de conhecimento e motivação relativo à saúde bucal nas duas escolas é bom, embora houvesse algumas diferenças estatisticamente significantes entre elas. Em relação à escovação dentária, as crianças da Escola 1 apresentaram maior conhecimento e motivação que as crianças da Escola 2, resultados da orientação recebida de seus pais. Verificou-se também que, apesar de darem muito valor aos dentes, as crianças de ambas escolas ainda apresentam folta de informações quanto ao uso correto do fio dental e técnicas de escovação mais adequadas, o que justificaria a implantação de programas de saúde pública odontológica tanto nas escolas públicas quanto nas particulares.

PALAVRAS-CHAVE: Saúde bucal; Motivação; Prevenção.

* Autores: Alunos do $5^{\circ}$ ano de Graduação em Odontologia da Universidade Estadual de Londrira.

at: Orientador: Prof. Adjunto de Odontologia Social e Preventiva da Universidade Estadual de Londrina/Centro de Ciências da Saúde- rua Pernamiuco, j4) Fone (043) 321-2m12 - Cep 86020-070 - Lontrina - Paraní. 
ABSTRACT: The present study is a field-work to evaluate the knowledge level and motivation related to the oral health in children from two schools of Londrina, State of Paraná: Colégio Universitário (School 1) and Colégio Estadual Olympia M. de Tormenta (School 2). In this opportunity it was analysed, in each school, the importance that children give to oral health and the information level about the hygiene and the ways of dental caries prevention. The data were collected through questionnaires applied to 168 students of both sexes, 86 in each school, with age ranging from 11 to 15 years old (mean $=12$ years old). The analyses of the results obtained permitted to conclude that the knowledge level and motivation related to the oral health in two schools is good, although some differences, statistically significant, were detected. In regard to dental toothbrushing, children from school 1 presented more knowledge and motivation than children from school 2, resulted by the guidance received from their parents. It was also verified that, although they had given value to the teeth, children from both schools already presented lack of information about the correct use of the dental floss dental and toothbrushing. Therefore, techniques more suitable would justify the introduction of public dental health progranis in both public schools and private schools.

KEYWORDS: Oral health; Motivation; Prevention.

\section{INTRODUÇÃO}

Diversos estudos e pesquisas comprovam que a cárie dentária e a docnça periodontal são passíveis de prevenção. A etiologia dessas enfermiciades já é bem conhecida c medidas preventivas eficazes já estão sendo usadas adequadamente (ALEXON, 1981).

Segundo CHUJF et al. (1984), a motivação por parte do profissional e a conscientização do paciente dos meios disponíveis para o controle da placa bacteriana são de fundamental importância para o êxito de qualquer tratamento odontológico.

A correta higiene oral, no seu sentido estrito de limpeza das superfícies dentárias, é considerada como um meio auxiliar eficaz na prevenção dos dois grupos mais importantes de enfermidade que prevalecem na cavidade oral : a cárie dentária e as enfermidades periodontais (SLACK, 1958 e CHAVES, 1960).

Segundo TOLEDO (1965), há neeessidade de desenvolvimento de programas de saúde pública dentária onde seja salientada a importância da higiene oral realizada $\mathrm{cm}$ casa, pois somente assim é possível obter resultados mais efetivos quanto à saúde bucal.
Segundo SANDELL (1959), educação para saúde bucal é o processo de provocar uma mudança no indivíduo quanto ao seu comportamento relativo à saúde. Isto implica em um trabalho de conscientização do mesmo quanto à importância, visando torná-lo motivado e receptivo a estas mudanças.

Nessc contexto, a educação para a saúde bucal deve ser enfatizada tanto em programas públicos de cducação coletiva, como individualmente no consultório (CASTELLANOS,1977), objetivando prover o indivíduo de informações necessárias ao desenvolvimento de hábitos de manutenção da saúde e prevenir contra as doenças bucais de maior prevalência entre as populações, a cárie e doença periodontal.

Assim, ao se verificar a grande importância da higiene oral como meio preventivo da cárie dentária e da doença periodontal, propôs-se a realização de um estudo para se avaliar o nível de conhecimento e motivação quanto a saúde bucal em crianças de duas escolas de Londrina, com características sociais, culturais e econômicas distintas. 


\section{2- MATERIAL E MÉTODOS}

2.1- MATERIAL: A população utilizada para este estudo compreendeu 186 crianças matriculadas na $6 \mathrm{a}$ série do ensino de 1 grau, sendo 73 do sexo masculino e 95 do sexo feminino, com idade variando de 11 a 15 anos (média de idade $=12$ anos), distribuídas em 2 escolas da cidade de Londrina, Paraná: 84 crianças do "Colégio Universitário", localizada no centro da cidade, na Rua Alagoas, 2001 (Escola 1) e 84 crianças do "Colégio Estadual Olympia M. de Tormenta", localizada na periferia da cidade, na Rua Keihold, 173 (Escola 2).

Tomou-se como amostra alunos da 6a série, onde prevaleciam crianças de 12 anos de idade, por ser esta idade padronizada pela OMS na verificação do índice de CPOD como indicador básico de comparação para o estado de saúde bucal entre populações diversas (OMS, 1985).

Os dados relativos à raça e nacionalidade não foram avaliados por não fazerem parte do objetivo do trabalho em questão.

Os dados relativos aos níveis sociais e econômicos dos pais dos escolares não estavam disponíveis. No cntanto, segundo informações obtidas na direção de cada escola, a Escola 1 apresenta padrões sócio-culturais e econômicos e nível de escolaridade dos pais mais elevados que a Escola 2.

2.2- MÉTODOS: Foi elaborado um questionário com 10 perguntas objetivas contendo 3 alternativas de resposta em cada uma delas, objetivando obter informações sobre o gradiente de conhecimento e motivação quanto à saúde bucal dos escolares selecionados. Os questionários foram aplicados individualmente, sempre conduzido pelo mesmo examinador. Os dados obtidos foram analisados estatisticamente através do Teste de qui-quadrado, ao nível de significância de $5 \%$.

\section{3- RESULTADOS}

As Tabelas 1, 2, 3, 4, 5 e 6 apresentam os resultados relacionados à caracterização das crianças quanto a faixa etária, freqüência de escovação dental, início do interesse da criança pela saúde bucal, dificuldades na escovação, conhecimento dos meios de prevenção da cárie e conhecimento quanto a relação entre o açúcar e a cárie dentária, respectivamente.

TABELA 1- Caracterização quanto a faixa etária das crianças analisadas nas duas escolas de Londrina, Paraná.

\begin{tabular}{|c|c|c|c|c|c|c|}
\hline \multirow[t]{3}{*}{ ESCOLA } & \multicolumn{5}{|c|}{ IDADE (ANOS) } & \multirow[t]{3}{*}{ TOTAL } \\
\hline & 11 & 12 & 13 & 14 & 15 & \\
\hline & П $\quad(\%)$ & n $(\%)$ & п $(\%)$ & n $\quad(\%)$ & П $(\%)$ & \\
\hline ESCOLA 1 & $9(10,71)$ & $68(80,95)$ & $7 \quad(8,33)$ & $0(0,00)$ & $0(0,00)$ & 84 \\
\hline ESCOLA 2 & $2(10,71)$ & $50(80,95)$ & $21(25,00)$ & $8 \quad(9,52)$ & $3(3,57)$ & 84 \\
\hline
\end{tabular}

TABELA 2- Freqüência de escovação dental diária nas crianças analisadas nas duas escolas de Londrina, Paraná

\begin{tabular}{|c|c|c|c|c|c|}
\hline \multirow{2}{*}{$\begin{array}{l}\text { FREQUÊNCIA } \\
\text { DE ESCOVAÇÃO }\end{array}$} & \multicolumn{2}{|c|}{ ESCOLA 1} & \multicolumn{2}{|c|}{ ESCOLA 2} & \multirow[t]{2}{*}{ TOTAL } \\
\hline & 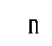 & $(\%)$ & $n$ & $(\%)$ & \\
\hline NÄO ESCOVA & 0 & $(0,00)$ & 0 & $(0,00)$ & 0 \\
\hline 2 VEZES OU MENOS & 13 & $(15,48)$ & 32 & $(38,10)$ & 45 \\
\hline 3 VEZES OU MAIS & 71 & $(84,52)$ & 52 & $(61,90)$ & 123 \\
\hline
\end{tabular}

TESTE QUI - QUADRADO, $\mathrm{p}=0,001$

TABELA 3- Início do interesse pela saúde bucal das crianças analisadas nas duas escolas de Londrina, Paraná

\begin{tabular}{llll}
\hline INÍCIO & ESCOLA 1 & ESCOLA 2 & TOTAL \\
DO INTERESSE & &
\end{tabular}

\begin{tabular}{|c|c|c|c|c|c|}
\hline & & & & & \\
\hline & $\mathrm{n}$ & $(\%)$ & n & $(\%)$ & \\
\hline COM OS PAIS & 72 & $(85,71)$ & 55 & $(65,48)$ & 127 \\
\hline COM O DENTISTA & 12 & $(14,29)$ & 24 & $(28,57)$ & 36 \\
\hline COM PROFESSOR & 0 & $(0,00)$ & 5 & $(5,95)$ & 5 \\
\hline
\end{tabular}


TABELA 4- Dificuldade na realização da escovação dental pelas crianças analisadas nas duas escolas de Londrina, PR.

\begin{tabular}{|c|c|c|c|}
\hline \multirow[t]{2}{*}{ DIFICULDADES } & ESCOLA 1 & ESCOLA 2 & TOTAL \\
\hline & $(\%)$ & $(\%)$ & \\
\hline
\end{tabular}

\begin{tabular}{lccccc} 
MUITO DIFÍCIL & 72 & $(85,71)$ & 55 & $(65,48)$ & 127 \\
\hline SEM TEMPO & & & & & \\
DISPONÍVEL & 19 & $(22,89)$ & 19 & $(23,17)$ & 38 \\
\hline SEM DISPOSIÇÃO & 37 & $(44,58)$ & 9 & $(10,98)$ & 46 \\
\hline
\end{tabular}

[ESTEQUI-QUADRADO, $p=0,001$

TABELA 5- Meios de prevenção da cárie dentária conhecidos p[elas crianças analisadas nas duas escolas de Londrina, PR.

\begin{tabular}{|c|c|c|c|c|c|}
\hline \multirow{2}{*}{$\begin{array}{l}\text { MEIOS DE } \\
\text { PREVENÇÃO }\end{array}$} & \multicolumn{2}{|c|}{ ESCOLA 1} & \multicolumn{2}{|c|}{ ESCOLA 2} & \multirow[t]{2}{*}{ TOTAL } \\
\hline & n & $(\%)$ & $\pi$ & $(\%)$ & \\
\hline ESCOVA+PASTA & 2 & $(2,38)$ & 5 & $(5,95)$ & 7 \\
\hline $\begin{array}{l}\text { ESCOVA+PASTA } \\
+\mathrm{FIO}\end{array}$ & 16 & $(19,05)$ & 4 & $(4,76)$ & 20 \\
\hline $\begin{array}{l}\text { ESCOVA+PASTA } \\
\text { FlO+FLÚOR }\end{array}$ & 66 & $(78,57)$ & 75 & $(89,29)$ & 141 \\
\hline
\end{tabular}

TABELA 6. Conhecimento quanto à relação entre o açúcar e a cáric dentária das crianças analisadas nas duas escolas de Londrina, Paraná.

\begin{tabular}{|c|c|c|c|c|c|}
\hline \multirow{2}{*}{$\begin{array}{l}\text { RELAÇÃO ENTRE } \\
\text { O AÇÚCAR E CÁRIE }\end{array}$} & \multicolumn{2}{|c|}{ ESCOLA 1} & \multicolumn{2}{|c|}{ ESCOLA 2} & \multirow[t]{2}{*}{ TOTAL } \\
\hline & $\mathrm{n}$ & $(\%)$ & $n$ & $(\%)$ & \\
\hline NÃO DEVE & & & & & \\
\hline CONSUMIR AÇUCAR & 4 & $(4,76)$ & 22 & $(26,19)$ & 26 \\
\hline $\begin{array}{l}\text { AÇÚCAR NÃO } \\
\text { INTERFERE NA } \\
\text { CÁRIE }\end{array}$ & 2 & $(2,38)$ & 4 & $(4,76)$ & 6 \\
\hline $\begin{array}{l}\text { AÇÚCAR É NOCIVO } \\
\text { MAS PODE SER } \\
\text { CONSUMIDO DE } \\
\text { FORMA EQUILIBRAD }\end{array}$ & 78 & $(92,86)$ & 58 & $(69,05)$ & 139 \\
\hline
\end{tabular}

As Figuras 1, 2, 3 e 4 apresentam os resultados relacionados à caracterização das crianças quanto ao sexo, importância dada aos dentes, freqüência do uso do fio dental e dificuldades no uso do fio dental, respectivamente
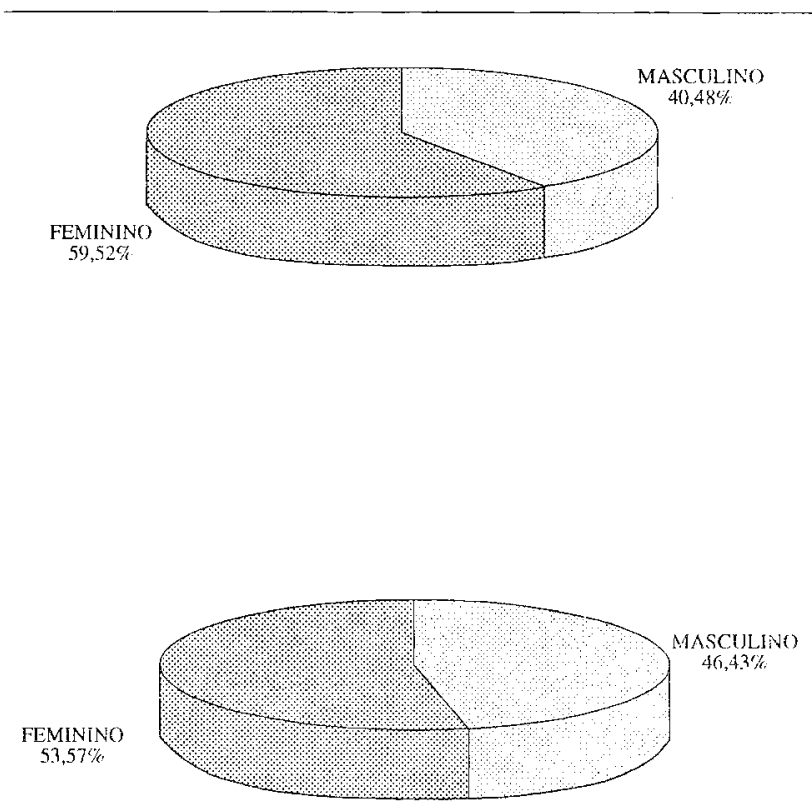

FHGURA A $\mathbb{1}$ - Caracterização quanto ao sexo das crianças analisadas nas duas escolas de Londrina, Paraná.
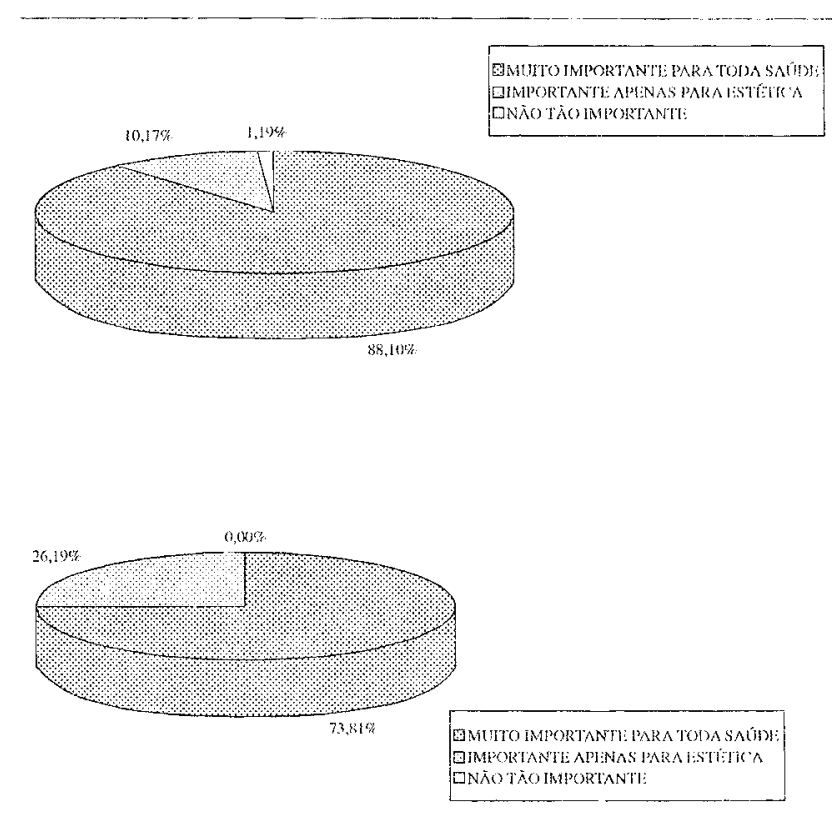

FIGURA 2-Importância dos dentes, segundo a crianças analisadas nas duas escolas de Londrina, Paraná 


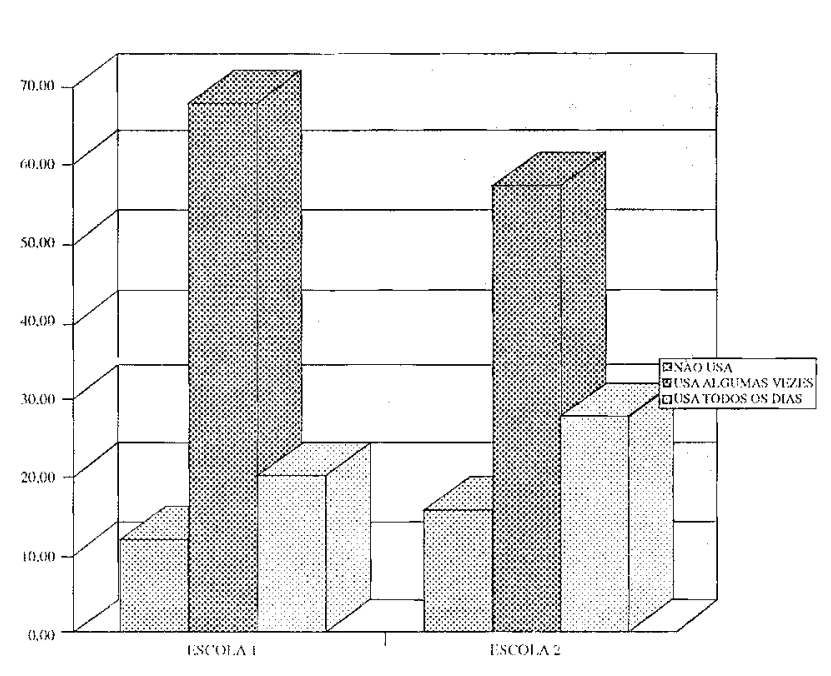

FIGURA 3 - Frequência do uso do fío dental pelas crianças analisadas nas duas escolas de Londrina, Paraná.

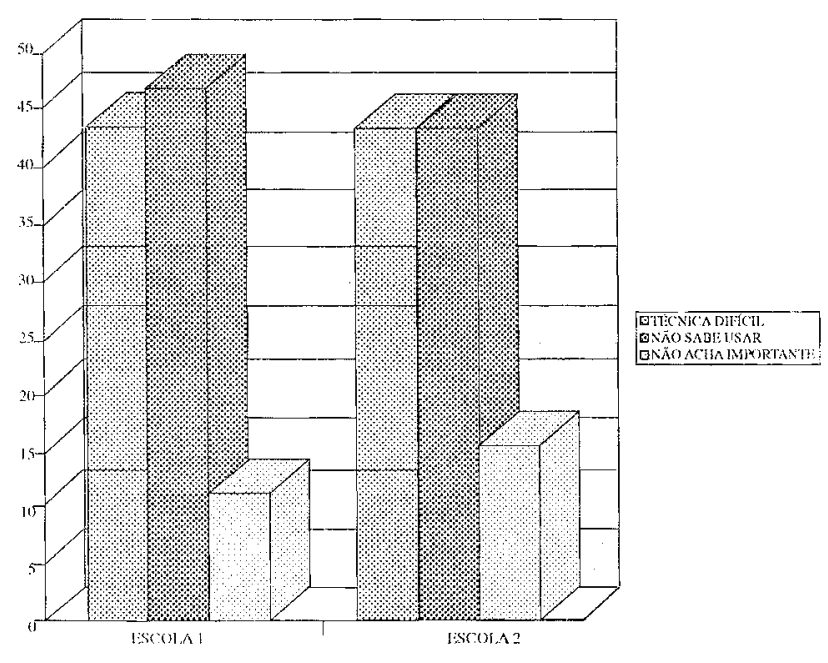

FIGURA 4 - Dificuldades encontradas no uso do fio dental pelas crianças analisadas nas duas escolas de Londrina, Paraná.

\section{DISCUSSÃO}

A média de idade das crianças das duas escolas foi de 12 anos e a distribuição quanto ao sexo foi homogênea conforme demonstra tabela 1 e figura 1.

Nas duas escolas, a maioria das crianças pesquisadas escova os seus dentes com uma frequiência diária de 3 vezes ou mais. No entanto, houve difcrenças estatisticamente significantes entre elas $(p=0,001)$. Verificou-se que as crianças da Escola 1 escovam mais os seus dentes que as da Escola 2 (Tabela 2).

O fato dos pais apresentarem um papel importante na boa higiene oral de seus filhos (TOLEDO, 1965), poderia explicar esta diferença na freqüência de escovação nas duas escolas, pois na Escola 1 os pais mostram-se mais participativos, quanto ao interesse pela saúde bucal de seus filhos, comparado à Escola 2, sendo $85,71 \%$ (Escola 1) e $65,48 \%$ (Escola 2), como demonstra a Tabela 3.

Esta diferente participação dos pais nas duas escolas, refletiu-se também quanto às barreiras encontradas para a realização de uma boa higiene oral, onde se encontrou também diferenças estatisticamente significantes ( $\mathrm{p}=$ 0,001). De acordo com a Tabela 4, para as crianças da Escola 1, a maior barreira está na falta de disposição, enquanto que para as crianças da Escola 2 está na técnica de escovação realizada, mostrando com isso, que não tiveram orientações adequadas en casa com os pais.

Apesar do fio dental ser o método de limpeza das superfícies proximais mais comumente recomendado (MOHAMMED, 1965), verificou-se que, na população estudada, a frequiência quanto ao seu uso é insuficiente, uma vez que somente 20,24\% das crianças da Escola 1 e $27,38 \%$ da Escola 2 responderam usá-1o diariamente (Figura 3). De acordo com VIEGAS (1971), a escovação por si só não consegue remover toda a placa dentária, principalmente aquela acumulada nas faces proximais dos dentes, fazendo-se necessário o uso do fio dental com a mesma freqüência de escovação.

Além da falta de motivação e insuficiente informação quanto à importância do fio dental como um meio complementar essencial da escovação dentária para limpeza completa dos dentes, verificou-se também a falta de orientação quanto ao modo de usá-jo (Figura 4); 
$46,43 \%$ das crianças da Escola 1 e $42,68 \%$ da Escola 2 responderam não saber usar o fio dental, e além disso $42,56 \%$ das crianças da Escola 1 e 42,68\% da Escola 2 consideram a técnica difícil para o seu uso. Segundo ROBSON (1976), o fio dental, ao ser empregado sem a técnica adcquada e apressadamente, não melhora os efeitos da escovação.

Embora não significante estatisticamente, as crianças das duas escolas apresentaram diferença quanto ao conhecimento de como prevenir a cárie dentária. Através deste estudo, demonstrado pela Tabela 5, verificou-se que $78,57 \%$ (Escola 1) e 89,29\% (Escola 2) responderam que além da escovação + pasta + fio dental, o flúor é também um meio de prevenção da cárie dentária. Esta diferença, apesar de ser pequena, poderia ser explicada pelo trabalho de aplicação de flúor através de bochecho ser rotineiramente realizado somente nas cscolas públicas (municipais e estaduais), não abrangendo as escolas particulares. Scgundo NEWBRUN (1988), os programas de bochechos com flúor nas escolas, realizados semanalmente con $0,2 \%$ de fluoreto de sódio durante 1 minuto, apresentam redução de cárie de 20 a $40 \%$.

Ao investigarmos a relação entre o açúcar e a cárie dentária, verificou-se que a maioria das crianças apresentou bom conhecimento sobre o assunto, pois 92,86\% (Escola 1) e 69,05\% (Escola 2) responderam ter conhecimento do cfeito nocivo do açúcar em relação à cáric, c que seu consumo poderia ser realizado, desde que fosse de forma equilibrada (Tabela 6). Segundo trabalho realizado por VIEGAS (1966), há uma correlação positiva entre o número de ingestões de alimentos açucarados e a cárie dental, com um CPO mais alto entre as crianças com três ou mais ingestões diárias freqüentemente efetuadas nos intervalos das principais refeições.

Apesar dos dentes serem considerados muito importantes para loda a saúde do individuo (Figua 2), as informações sobre medidas preventivas, como o uso adequado do fĩo dental c técnica de escovação, não estão sendo bem transmilidas, como mostraram os resultados obtidos nesta pesquisa.

\section{CONCLUSÕES}

Levando-se em consideração as limitações presentes neste trabalho, algumas conclusões podem ser destacadas:

1 - o nível de escolaridade dos pais poderia ser um fator que interfere na motivação e orientação das crianças quanto a higiene oral;

2- o nível de conhecimento e motivação relativo à saúde bucal das crianças pesquisadas foi bom, entre eles o nível de conhecimento sobre o papel do flúor como um meio de prevenção da cárie dentária $(78,57 \%$ na Escola $j$ e $89,89 \%$ na Escola 2) e o nível de conhecimento sobre o efeito nocivo do açúcar $(92,96 \%$ na Escola 1 e $69,05 \%$ na Escola 2);

3- houve faíta de informações adcquadas quanto ao uso do fio dental e a técnica de escovação dentária.

Estes fatos vêm relorçar a importância dos programas de saúde pública, tanto nas escolas públicas como nas particulares, na educação e prevenção da saúde bucal.

\section{AOMADEOMMENTOS}

- A Profa Edna Maria Vissoci Rciche, pelo valioso apoio e ensmamentos, sem os quais não seria possíve? a realização deste trabalho.

- A Profa Dra. Tiemi Matsuo, pelo empenho com que conduziu as análises esiatústicas.

- Ao amigo José Tadashi Tanaka pela sua dcoicação na confecção dos gráficos e das tabelas.

- À Profa Maisa Christina Nascimento de Almeida pela sua valiosa colaboração quanto às traduções realizadas no trabalho. 


\section{REFERÊNCIAS BIBLIOGRÁFICAS}

01. ALEXON, P. I- A odontologia preventiva deve basear-se no controle de placa bacteriana. IIEstabelecimento de hábitos de higiene bucal dirigido pela localização de placas e de doença dentária. Tradução de Maria Inês Beidi e Hamilton Taddel Bellini, São Paulo, Faculdade de Odontologia da USP, 1981, 63p.

02. CASTELLANOS, R. A. Orientação sobre saúde bucal em um centro de saúde. Rev. Saúde Pública, v.11, n.2, p. 248-257, 1977.

03. CHAVE, M. M. Necessidade de uma consciência preventiva e sanitária no profissional. Rev. Un. Odont. Bras., São Paulo v. 1, p. 47, mar / abril, 1960.

04. CHUJFI, E. S. et a1. Conceito atual e algumas considerações sobre os métodos de afastamento gengival sobre as estruturas dentais. Odont. Mod., v.11, n.6, p. 17-22, jun, 1984.

05. MOHAMMED, C. Dental plaque removed by floss. J. New Jersey Dent. Soc., v. 36, p. 419, 1965.

06. NEWBRUN, E. Cariologia. 2.ed. São Paulo: Editora Santos, 1988.

07. OMS. Oral dental health global indicator for 2000: DMTF - dental cáries levels at 12 years, Geneva.

08. PINTO, V.G. Saúde bucal: odontologia social e preventiva. 3. ed. São Paulo: Editora Santos, 1992.

09. ROBINSON, $\mathbb{E}$. A comparative evaluation of the Srub and Bass methods of toothbrushing with flossing as on adjunct. Am. J. Pub. Helih, v. 66, p. 1078 - 81, nov, 1976.

10. SANDELL, P. Health education activities in community dental programa. J. Dent. Child. v. 26, n. 3, p. 224-228, 1959.

11. SLACK, G.L. Dental disease - the last great epidemic (symposium) R.H.S., v. 4, p. 476, 1958.
12. TOLEDO, B.E.C. Avaliação do estado de orientação da higiene oral em escolares da cidade de Araraquara. Rev. Assoc. Paulista de cirurgiôes dentistas, v. 19, n. 6, p. 217-230, nov / dez, 1965.

13. VIEGAS, A. R. Relação entre o número de ingestões de alimentos açucarados e a cárie dental. Separata de arquivos da Faculdade de higiene e saúde pública da Universidade de São Paulo, v. 20, n. 2, p.155-166, dez, 1966.

14. VIEGAS, A. R. Prevenção da cárie dental. In: Encontro de Saúde Pública em Odontologia, 2, Porto Alegre, 1971. Anais...Porto Alegre, PMPA. 1971. 